JOURNAL OF INTEGRAL EQUATIONS

AND APPLICATIONS

Volume 4, Number 1, Winter 1992

\title{
THE NUMERICAL APPROXIMATION OF THE SOLUTION OF A NONLINEAR BOUNDARY INTEGRAL EQUATION WITH THE COLLOCATION METHOD
}

\author{
M. HAMINA, K. RUOTSALAINEN AND J. SARANEN
}

\begin{abstract}
Recently, Galerkin and collocation methods have been analyzed in connection with the nonlinear boundary integral equation which arises in solving the potential problem with a nonlinear boundary condition. Considering this model equation, we propose here a discretized scheme such that the nonlinearity is replaced by its $L^{2}$-orthogonal projection. We are able to show that this approximate collocation scheme preserves the theoretical $L^{2}$-convergence. For piecewise linear continuous splines, our numerical experiments confirm the theoretical quadratic $L^{2}$-convergence.
\end{abstract}

1. Introduction. We consider the solution of the potential equation in a bounded domain $\Omega$ with a given Neumann-type nonlinear boundary condition. Taking the model problem of $[\mathbf{1 2}, \mathbf{1 3}]$, consider

$$
\begin{cases}\Delta \Phi=0, & \text { in } \Omega \\ -\left.\partial_{n} \Phi\right|_{\Gamma}=f(x, \Phi)-g, & \text { on } \Gamma=\partial \Omega .\end{cases}
$$

We assume that the boundary $\Gamma$ is a smooth Jordan-curve in the plane. Conditions for the nonlinear function $f(x, \Phi)$ as well as for the given boundary data $g$ will be specified later.

By using Green's representation formula for the potential $\Phi$, problem (1.1) reduces to the following nonlinear boundary integral equation [13]

$$
\frac{1}{2} u-K u+V F(u)=V g .
$$

Here $V$ is the single layer boundary integral operator

$$
V u(x):=\frac{-1}{2 \pi} \int_{\Gamma} u(y) \ln |x-y| d s_{y},
$$

1980 Mathematics Subject Classification: 65N35, 45L10, 35J65, 31A10.

Key words and phrases. Collocation, boundary integrals, nonlinear, potential.

Copyright (c)1992 Rocky Mountain Mathematics Consortium 
$K$ is the double layer boundary integral operator

$$
K u(x):=\frac{1}{2 \pi} \int_{\Gamma} u(y) \frac{\partial}{\partial n_{y}} \ln |x-y| d s_{y},
$$

and $u$ is the boundary density. Ruotsalainen and Wendland [13] discuss the solvability of the equation (1.2) and analyze the convergence of the spline Galerkin approximation method. Another theoretical approach for the analysis of the numerical schemes, covering also the spline collocation method, was presented by Ruotsalainen and Saranen [12]. In addition, Atkinson and Chandler proposed [4] two other numerical approaches for problem (1.1), namely the use of the Nyström Method and a method based on trigonometric interpolation. Based on the framework of monotone operators, $[\mathbf{1 2}, \mathbf{1 3}]$ give optimal order convergence results, if the approximation error of the boundary density $u$ is measured by the Sobolev norm of order 1/2. Later, Saranen [14] was able to prove the optimal $L^{2}$-convergence. From a practical point of view, the collocation method is superior to the Galerkin method. However, in the actual numerical implementation, the nonlinearity must be handled carefully in order to retain the convergence properties. The purpose of our paper is to introduce an approximation scheme for (1.2) by using an easily computable $L^{2}$-orthogonal projection of the nonlinear function. This approach applies to general projection methods, but for simplicity, we discuss only collocation. It turns out that our method retains the optimal convergence order of the collocation method. We also take into account the effect of numerical integration in the scheme. Numerical experiments confirm our theoretical results.

2. Preliminaries. We assume that the boundary $\Gamma$ has a regular 1-periodic parameterization $x(t): \mathbf{R} \rightarrow \Gamma$ such that $|d x / d t| \geq \rho_{0}>0$. Let $\Delta_{h}=\left\{x_{k}=x\left(t_{k}\right) \mid 0=t_{0}<\cdots<t_{N}=1\right\}, h=1 / N$, be a mesh on $\Gamma$, and let $S_{h}^{d}$ be the corresponding space of smoothest splines of degree $d \geq 0$ on the periodic partition $\left\{t_{k} \mid k \in \mathbf{Z}\right\} \subset \mathbf{R}$. We assume that the family of partitions $\left\{\Delta_{h} \mid h>0\right\}$ is quasiuniform.

In the following, $H^{s}(\Gamma)$, where $s \in \mathbf{R}$, denotes the usual Sobolev space equipped with the norm $\|u\|_{s}=(u \mid u)_{s}^{1 / 2}$. In particular, we have $H^{0}(\Gamma)=L^{2}(\Gamma)$ and $(u \mid v)_{0}=\int_{\Gamma} u(x) v(x) d s_{x}$. We frequently identify the 1-periodic function $u(x(t))$ with the function $u(x)$ defined on the boundary $\Gamma$. 
The following approximation and inverse properties are well known $[2,7]$.

Approximation Property. Let $t \leq s \leq d+1, t<d+1 / 2$. Then, for all $u \in H^{s}(\Gamma)$, there exists $\phi \in S_{h}^{d}$ such that

$$
\|u-\phi\|_{t} \leq c h^{s-t}\|u\|_{s},
$$

where the constant $c$ is independent of $u$ and $h$.

Inverse Property. Let $t \leq s<d+1 / 2$. Then there exists a constant $c$ independent of $h$ such that

$$
\|\phi\|_{s} \leq c h^{t-s}\|\phi\|_{t}
$$

for all $\phi \in S_{h}^{d}$.

The real valued nonlinear function $f(\cdot, \cdot): \Gamma \times \mathbf{R} \rightarrow \mathbf{R}$ is assumed to satisfy the Carathéodory conditions

$$
\begin{gathered}
f(\cdot, u): \Gamma \rightarrow \mathbf{R} \text { is measurable for all fixed } u \in \mathbf{R} \\
f(x, \cdot): \mathbf{R} \rightarrow \mathbf{R} \text { is continuous for almost all } x \in \Gamma .
\end{gathered}
$$

The associated Nemitsky operator is defined by

$$
F(u)(x)=f(x, u(x))
$$

The Nemitsky operator $u \mapsto F(u)$ is a well-defined operator from $L^{2}(\Gamma)$ to $L^{2}(\Gamma)$ if the Carathéodory conditions and the growth condition $|f(x, u)| \leq a(x)+b(x)|u|$, are valid [9]. For the analysis of the numerical approximation scheme, we make the supplementary assumption:

A1. The Nemitsky operator $F$ is strongly monotone, i.e., for every $w, u \in L^{2}(\Gamma)$,

$$
(F(u)-F(w) \mid u-w)_{0} \geq c|| u-w \|_{0}^{2} .
$$

A2. The Carathéodory function $f(\cdot, \cdot)$ is such that $F: L^{2}(\Gamma) \rightarrow L^{2}(\Gamma)$ is Lipschitz continuous. 
A3. The Nemitsky operator $F: H^{s}(\Gamma) \rightarrow H^{s}(\Gamma)$ is bounded for all $0 \leq s<1$.

We remark that (A2) and (A3) are valid if $f$ is Lipschitz continuous.

The mapping properties of the operators $V$ and $K$ are quoted from [8] and we collect them in the following theorem. The capacity of the boundary $\Gamma$ is denoted by cap $(\Gamma)$.

Theorem 2.1. (1) If $\operatorname{cap}(\Gamma) \neq 1$, then $V: H^{s}(\Gamma) \rightarrow H^{s+1}(\Gamma)$ is an isomorphism.

(2) If $\operatorname{diam}(\Omega)<1$, then there exists $\sigma_{0}>0$ such that

$$
(V \psi \mid \psi)_{0} \geq \sigma_{0}\|\psi\|_{-\frac{1}{2}}^{2}, \quad \text { for all } \psi \in H^{-\frac{1}{2}}(\Gamma) .
$$

(3) $K: H^{s}(\Gamma) \rightarrow H^{s+1}(\Gamma)$ is continuous for all $s$.

The solvability of (1.2) and the regularity of the solution has been discussed in $[\mathbf{1 3}]$.

Theorem 2.2. Let cap $(\Gamma) \neq 1$.

(1) For every $g \in H^{-1 / 2}(\Gamma)$, the integral equation (1.2) has a unique solution $u \in H^{1 / 2}(\Gamma)$.

(2) For the solution $u$, the following regularity result is true: If $g \in H^{s-1}(\Gamma), 1 / 2 \leq s<2$, and assumptions (A1), (A2) and (A3) are valid, then the solution satisfies $u \in H^{s}(\Gamma)$.

The proof of this theorem is presented in [13]. It is based on the fact that the integral operator defined by

$$
A(w):=\left(\frac{1}{2} I-K\right) w+V F(w)
$$

is strongly $V^{-1}$-monotone, i.e., for all $u, w \in H^{1 / 2}(\Gamma)$, we have

$$
\left(A(u)-A(w) \mid V^{-1}(u-w)\right)_{0} \geq c\|u-w\|_{\frac{1}{2}}^{2} .
$$

In [13], the assumption $\operatorname{diam}(\Omega)<1$ was used. However, the assumption $\operatorname{cap}(\Gamma) \neq 1$ is sufficient. The proof $[\mathbf{1 3}]$ is still applicable. 
3. The collocation approximation. Let us now consider the collocation method for finding an approximate solution of the equation (1.2). We require that $g \in H^{s-1}(\Gamma), s>1 / 2$. Then the function $V g$ is continuous, and the collocation equations are given by: Find $u_{h} \in S_{h}^{d}$ such that

$$
A u_{h}\left(\tilde{x}_{i}\right)=\operatorname{Vg}\left(\tilde{x}_{i}\right), \quad i=0, \ldots, N-1
$$

where

$$
\begin{aligned}
& \tilde{x}_{i}=x\left(t_{i}\right), \quad d \text { is odd } \\
& \tilde{x}_{i}=x\left(\frac{t_{i}+t_{i+1}}{2}\right), \quad d \text { is even. }
\end{aligned}
$$

For the midpoint collocation, we assume that the mesh is smoothly graded in the sense of [3]. An equivalent formulation of equation (3.1) is: Find $u_{h} \in S_{h}^{d}$ such that

$$
I_{h} A u_{h}=I_{h} V g
$$

where the interpolation operator $I_{h}: H^{s}(\Gamma) \rightarrow S_{h}^{d}$ is defined by

$$
I_{h} \psi\left(\tilde{x}_{i}\right)=\psi\left(\tilde{x}_{i}\right), \quad i=0, \ldots, N-1
$$

By our assumption, the interpolation error satisfies the estimate

$$
\begin{gathered}
\left\|I_{h} w-w\right\|_{t} \leq c h^{s-t}\|w\|_{s} \\
0 \leq t<d+1 / 2,1 / 2<s \leq d+1, t \leq s .
\end{gathered}
$$

We shall need the following result, known for the collocation method.

Theorem 3.1. Assume $d>0$. Let $u \in H^{s}(\Gamma), 1 / 2<s \leq d+1$, be the solution of (1.2) and suppose that (A1) and (A2) are valid. Then, for sufficiently small $h$, the collocation problem (3.1) admits a unique solution $u_{h}$. Moreover, we have the asymptotic error estimate

$$
\left\|u-u_{h}\right\|_{t} \leq c h^{s-t}\|u\|_{s}
$$

where $0 \leq t \leq s$ and $t<d+1 / 2$. 
The proof presented in [12] covers indices $1 / 2 \leq t \leq s$ and the recent results by Saranen [14] give (3.4) for $0 \leq t<1 / 2$.

For numerical purposes, we define an approximate collocation equation as follows: Find $\tilde{u}_{h} \in S_{h}^{d}$ such that

$$
\tilde{A}_{h}\left(\tilde{u}_{h}\right):=\frac{1}{2} \tilde{u}_{h}-I_{h} K \tilde{u}_{h}+I_{h} V P_{h} F\left(\tilde{u}_{h}\right)=I_{h} V g .
$$

Here $P_{h}: L^{2}(\Gamma) \rightarrow S_{h}^{d}$ is the orthogonal projection defined by equation

$$
\left(P_{h} w \mid \chi\right)_{0}=(w \mid \chi)_{0} \quad \forall \chi \in S_{h}^{d} .
$$

The orthogonal projection possesses the approximation property

$$
\begin{gathered}
\left\|P_{h} w-w\right\|_{t} \leq c h^{s-t}\|w\|_{s}, \\
-d-1 \leq t<d+\frac{1}{2},-d-\frac{1}{2}<s \leq d+1, t \leq s,
\end{gathered}
$$

([11], Corollary 4). Solvability of (3.5) as well as the error estimates are based on the following stability property.

Theorem 3.2. Let $d>0$. There exists a positive constant $c_{1}$ such that

$$
\left\|\tilde{A}_{h}(\chi)-\tilde{A}_{h}(\psi)\right\|_{\frac{1}{2}} \geq c_{1}\|\chi-\psi\|_{\frac{1}{2}},
$$

for all $\chi, \psi \in S_{h}^{d}$ when $0<h \leq h_{0}$. Moreover, equation (3.5) has a unique solution for $0<h \leq h_{0}$.

Proof. Since $d>0$, we have $S_{h}^{d} \subset H^{1 / 2}(\Gamma)$. For splines $\psi \in S_{h}^{d}$, we have

$$
\tilde{A}_{h}(\psi)=\frac{1}{2} \psi-I_{h} K \psi+I_{h} V P_{h} F(\psi) .
$$

The mapping properties of $K, V$ and the continuity of $F$ together with (3.3), (3.7) imply the continuity of $\tilde{A}_{h}: S_{h}^{d} \rightarrow S_{h}^{d}$.

Next we prove the stability estimate (3.8). We abbreviate

$$
\tilde{B}(\chi):=-K \chi+V P_{h} F(\chi) \quad B(\chi):=-K \chi+V F(\chi) .
$$


By the estimate

$$
\left(A(\chi)-A(\psi) \mid V^{-1}(\chi-\psi)\right)_{0} \geq c|| \chi-\left.\psi\right|_{\frac{1}{2}} ^{2}
$$

and Theorem 2.1, we have

$$
\begin{aligned}
&\left(\tilde{A}_{h}(\chi)-\tilde{A}_{h}(\psi) \mid V^{-1}(\chi-\psi)\right)_{0} \geq c\|\chi-\psi\|_{\frac{1}{2}}^{2} \\
& \quad-\left\|\left(I-I_{h}\right)(\tilde{B}(\chi)-\tilde{B}(\psi))\right\|_{\frac{1}{2}}\left\|V^{-1}(\chi-\psi)\right\|_{-\frac{1}{2}} \\
& \quad-\|(\tilde{B}-B)(\chi)-(\tilde{B}-B)(\psi)\|_{\frac{1}{2}}\left\|V^{-1}(\chi-\psi)\right\|_{-\frac{1}{2}} \\
& \geq\left\{c\|\chi-\psi\|_{\frac{1}{2}}-\left\|\left(I-I_{h}\right)(\tilde{B}(\chi)-\tilde{B}(\psi))\right\|_{\frac{1}{2}}\right. \\
&\left.\quad-\|(\tilde{B}-B)(\chi)-(\tilde{B}-B)(\psi)\|_{\frac{1}{2}}\right\}\left\|V^{-1}(\chi-\psi)\right\|_{-\frac{1}{2}}
\end{aligned}
$$

for all splines $\psi$ and $\chi$. Using the approximation property (3.3), we get $\left\|\left(I-I_{h}\right)(\tilde{B}(\chi)-\tilde{B}(\psi))\right\|_{\frac{1}{2}} \leq c h^{\frac{1}{2}}\left\{\|K(\chi-\psi)\|_{1}+\left\|V P_{h}(F(\chi)-F(\psi))\right\|_{1}\right\}$.

Since $K: H^{1 / 2}(\Gamma) \rightarrow H^{1}(\Gamma), V: L^{2}(\Gamma) \rightarrow H^{1}(\Gamma)$ and the orthogonal projection $P_{h}: L^{2}(\Gamma) \rightarrow L^{2}(\Gamma)$ are continuous and the Nemitsky operator $F: L^{2}(\Gamma) \rightarrow L^{2}(\Gamma)$ is Lipschitz continuous, we obtain

$$
\left\|\left(I-I_{h}\right)(\tilde{B}(\chi)-\tilde{B}(\psi))\right\|_{\frac{1}{2}} \leq \operatorname{ch}^{\frac{1}{2}}\|\chi-\psi\|_{\frac{1}{2}} .
$$

Similarly, by Theorem 2.1 and the approximation property (3.7), we have

$(3.12)$

$$
\begin{aligned}
\|(\tilde{B}-B)(\chi)-(\tilde{B}-B)(\psi)\|_{\frac{1}{2}} & \leq c\left\|\left(P_{h}-I\right)(F(\chi)-F(\psi))\right\|_{-\frac{1}{2}} \\
& \leq \operatorname{ch}^{\frac{1}{2}}\|F(\chi)-F(\psi)\|_{0} \\
& \leq \operatorname{ch}^{\frac{1}{2}}\|\chi-\psi\|_{\frac{1}{2}} .
\end{aligned}
$$

If the parameter $h_{0}$ is sufficiently small, then the estimates (3.10), (3.11) and (3.12) imply

(3.13) $\left(\tilde{A}_{h}(\chi)-\tilde{A}_{h}(\psi) \mid V^{-1}(\chi-\psi)\right)_{0} \geq c|| \chi-\psi\left\|_{\frac{1}{2}}\right\| V^{-1}(\chi-\psi) \|_{-\frac{1}{2}}$, for $0<h \leq h_{0}$. Now, the stability (3.8) follows from (3.13) by the Schwarz inequality

$$
\begin{aligned}
c\|\chi-\psi\|_{\frac{1}{2}}\left\|V^{-1}(\chi-\psi)\right\|_{-\frac{1}{2}} & \leq\left(\tilde{A}_{h}(\chi)-\tilde{A}_{h}(\psi) \mid V^{-1}(\chi-\psi)\right)_{0} \\
& \leq\left\|\tilde{A}_{h}(\chi)-\tilde{A}_{h}(\psi)\right\|_{\frac{1}{2}}\left\|V^{-1}(\chi-\psi)\right\|_{-\frac{1}{2}} .
\end{aligned}
$$


Due to the stability result (3.8), the operator $\tilde{A}_{h}$ is an injection. According to the Brouwer theorem on invariance of the domain $([6]$, Theorem 4.3 , p. 23$)$ the range $R\left(\tilde{A}_{h}\right)$ is open. On the other hand, the stability (3.8) and the continuity of $\tilde{A}_{h}$ imply that $R\left(\tilde{A}_{h}\right)$ is also closed. Thus, $R\left(\tilde{A}_{h}\right)=S_{h}^{d}$ and $\tilde{A}_{h}$ is a homeomorphism.

The final theorem of this section describes the convergence of the approximate collocation solution $\tilde{u}_{h}$.

Theorem 3.3. Assume $d>0$. Let $u \in H^{s}(\Gamma), 1 / 2<s \leq d+1$ be the solution of (1.2) and suppose that the assumptions (A1), (A2) are valid. Then we have the estimates

$$
\begin{gathered}
\left\|\tilde{u}_{h}-u_{h}\right\|_{\frac{1}{2}} \leq c h^{s+\frac{1}{2}}\|u\|_{s}+c h^{\tau+\frac{1}{2}}\|F(u)\|_{\tau} \\
\left\|u-\tilde{u}_{h}\right\|_{t} \leq c h^{s-t}\|u\|_{s}+c h^{\tau+1-\max \left(t, \frac{1}{2}\right)}\|F(u)\|_{\tau}
\end{gathered}
$$

for $0 \leq t \leq s, t<d+1 / 2$, provided that $F(u) \in H^{\tau}(\Gamma), 0 \leq \tau \leq d+1$.

Proof. Using the stability result $[\mathbf{1 2}]$

$$
\left\|\tilde{u}_{h}-u_{h}\right\|_{\frac{1}{2}} \leq c|| I_{h} A\left(\tilde{u}_{h}\right)-I_{h} A\left(u_{h}\right) \|_{\frac{1}{2}},
$$

the relation $\tilde{A}_{h}\left(\tilde{u}_{h}\right)=I_{h} A\left(u_{h}\right)$ and the approximation property of $I_{h}$, we are able to estimate

$$
\begin{aligned}
\left\|\tilde{u}_{h}-u_{h}\right\|_{\frac{1}{2}} & \leq c\left\|I_{h} A\left(\tilde{u}_{h}\right)-I_{h} A\left(u_{h}\right)\right\|_{\frac{1}{2}} \\
& =c\left\|I_{h} V\left(I-P_{h}\right) F\left(\tilde{u}_{h}\right)\right\|_{\frac{1}{2}} \\
& \leq c\left\|\left(I_{h}-I\right) V\left(I-P_{h}\right) F\left(\tilde{u}_{h}\right)\right\|_{\frac{1}{2}}+c\left\|V\left(I-P_{h}\right) F\left(\tilde{u}_{h}\right)\right\|_{\frac{1}{2}} \\
& \leq c h^{\frac{1}{2}}\left\|V\left(I-P_{h}\right) F\left(\tilde{u}_{h}\right)\right\|_{1}+c\left\|\left(I-P_{h}\right) F\left(\tilde{u}_{h}\right)\right\|_{-\frac{1}{2}} \\
& \leq c h^{\frac{1}{2}}\left\|\left(I-P_{h}\right) F\left(\tilde{u}_{h}\right)\right\|_{0}+c\left\|\left(I-P_{h}\right) F\left(\tilde{u}_{h}\right)\right\|_{-\frac{1}{2}} \\
& =: T_{1}+T_{2} .
\end{aligned}
$$

Here we have by (3.7)

$$
\begin{aligned}
T_{2} & =c\left\|\left(I-P_{h}\right)\left(I-P_{h}\right) F\left(\tilde{u}_{h}\right)\right\|_{-\frac{1}{2}} \\
& \leq c h^{\frac{1}{2}}\left\|\left(I-P_{h}\right) F\left(\tilde{u}_{h}\right)\right\|_{0} \equiv T_{1} .
\end{aligned}
$$


Since $I-P_{h}$ is bounded and $F$ is Lipschitz continuous in $L^{2}(\Gamma)$, we obtain

$$
\begin{aligned}
T_{1} \leq & \operatorname{ch}^{\frac{1}{2}}\left\{\left\|\left(I-P_{h}\right)\left(F\left(\tilde{u}_{h}\right)-F\left(u_{h}\right)\right)\right\|_{0}+\left\|\left(I-P_{h}\right)\left(F\left(u_{h}\right)-F(u)\right)\right\|_{0}\right. \\
& \left.+\left\|\left(I-P_{h}\right) F(u)\right\|_{0}\right\} \\
\leq & \operatorname{ch}^{\frac{1}{2}}\left\{\left\|F\left(\tilde{u}_{h}\right)-F\left(u_{h}\right)\right\|_{0}+\left\|F\left(u_{h}\right)-F(u)\right\|_{0}+\left\|\left(I-P_{h}\right) F(u)\right\|_{0}\right\} \\
\leq & \operatorname{ch}^{\frac{1}{2}}\left\{\left\|\tilde{u}_{h}-u_{h}\right\|_{0}+\left\|u_{h}-u\right\|_{0}+\left\|\left(I-P_{h}\right) F(u)\right\|_{0}\right\} \\
\leq & \operatorname{ch}^{\frac{1}{2}}\left\{\left\|\tilde{u}_{h}-u_{h}\right\|_{\frac{1}{2}}+\left\|u_{h}-u\right\|_{0}+\left\|\left(I-P_{h}\right) F(u)\right\|_{0}\right\} .
\end{aligned}
$$

The approximation property of the orthogonal projection $P_{h}$ and the convergence result for $u_{h}$ imply the estimate

$$
\left\|\tilde{u}_{h}-u_{h}\right\|_{\frac{1}{2}} \leq T_{1} \leq c h^{\frac{1}{2}}\left\|\tilde{u}_{h}-u_{h}\right\|_{\frac{1}{2}}+c h^{s+\frac{1}{2}}\|u\|_{s}+c h^{\tau+\frac{1}{2}}\|F(u)\|_{\tau} .
$$

The convergence estimate (3.14) is proved. Finally, Theorem 3.1 together with (3.14) and the inverse property (2.2) imply (3.15).

By (3.15), the rate of the convergence depends on the regularity of the solution $u$ and of the regularity of the function $F(u)$. But the regularity of $u$ and of $F(u)$ are related to each other. For example, if $u \in H^{s}(\Gamma), g \in H^{s-1}(\Gamma)$, we conclude from equation (1.2) by the mapping properties of $K$ and $V$, that $F(u) \in H^{s-1}(\Gamma)$ with

$$
\|F(u)\|_{s-1} \leq c\left(\|u\|_{s}+\|g\|_{s-1}\right) .
$$

Hence, we have

Corollary 3.1. Suppose that (A1) and (A2) are valid. Let $u \in H^{s}(\Gamma)$ be the solution of (1.2) and let $g \in H^{s-1}(\Gamma), 1 \leq s \leq d+2$. Then we have

$$
\begin{aligned}
& \left\|u-\tilde{u}_{h}\right\|_{t} \\
\leq & c h^{\min (s, d+1)-t}\|u\|_{\min (s, d+1)}+c h^{s-\max \left(t, \frac{1}{2}\right)}\left(\|u\|_{s}+\|g\|_{s-1}\right),
\end{aligned}
$$

for all $0 \leq t \leq s, t<d+1 / 2$. In particular, for the $L^{2}$-norm, we obtain

$$
\left\|u-\tilde{u}_{h}\right\|_{0} \leq c h^{d+1}\left(\|u\|_{d+\frac{3}{2}}+\|g\|_{d+\frac{1}{2}}\right) .
$$


On the other hand, assume that (A2) is valid. If only the regularity $g \in H^{s-1}(\Gamma)$ is known, we can still conclude that $u \in H^{s}(\Gamma), 1 / 2<$ $s \leq 1$. Thus, we obtain

Corollary 3.2. Suppose that (A1) and (A2) are valid. Let $u \in$ $H^{1 / 2}(\Gamma)$ be the solution of (1.2) and let $g \in H^{s-1}(\Gamma), 1 / 2<s \leq 1$. Then we have $u \in H^{s}(\Gamma)$ and

$$
\left\|u-\tilde{u}_{h}\right\|_{t} \leq c h^{s-t}\|u\|_{s}+c h^{1-\max \left(t, \frac{1}{2}\right)}\|F(u)\|_{0},
$$

for all $0 \leq t \leq s$.

In this case we obtain the following estimate

$$
\left\|u-\tilde{u}_{h}\right\|_{0} \leq \operatorname{ch}^{\frac{1}{2}}\left(\|u\|_{1}+\|g\|_{0}\right) .
$$

These estimates can be improved by assuming more smoothness on the Nemitsky operator, e.g., (A3).

4. A modified equation. Here we will slightly generalize our results. Decomposing the single layer operator $V$ into the principal part with logarithmic singularity and the smooth part, we write the equation (1.2) to an explicit form

$$
\begin{aligned}
\frac{1}{2} u(x(t)) & -\frac{1}{2 \pi} \int_{0}^{1} u(x(\tau)) n(\tau) \cdot \frac{(x(\tau)-x(t))}{|x(\tau)-x(t)|^{2}}\left|x^{\prime}(\tau)\right| d \tau \\
& -\frac{1}{2 \pi} \int_{0}^{1}(F(u))(x(\tau)) \ln \frac{|x(t)-x(\tau)|}{|\tau-t|_{*}}\left|x^{\prime}(\tau)\right| d \tau \\
& -\frac{1}{2 \pi} \int_{0}^{1}(F(u))(x(\tau)) \ln |t-\tau|_{*}\left|x^{\prime}(\tau)\right| d \tau=V g(x(t)) .
\end{aligned}
$$

Here the modified distance defined by

$$
|t-\tau|_{*}=\min (|t-\tau|,|t-\tau+1|,|t-\tau-1|)
$$

makes the kernel

$$
\ln \frac{|x(t)-x(\tau)|}{|\tau-t|_{*}}
$$


a smooth function. We approximate with splines the product $v(t)=$ $u(x(t)) \kappa(t)$, where $\kappa(t):=\left|x^{\prime}(t)\right|$ is the Jacobian of the parametric representation. This computational method is frequently used since the integrals corresponding to the logarithmic singularity can be integrated exactly. Thus, numerical integration is needed only for the remaining part with a smooth kernel.

Writing (1.2) in terms of $v$, we obtain

$$
\frac{1}{2} v-\kappa K \frac{1}{\kappa} v+\kappa V F \frac{1}{\kappa}(v)=\kappa V g .
$$

We define the operator

$$
A_{\kappa}(v):=\kappa A \frac{1}{\kappa}(v)=\frac{1}{2} v-\kappa K \frac{1}{\kappa} v+(\kappa V \kappa)\left(\frac{1}{\kappa} F \frac{1}{\kappa}\right)(v) .
$$

Denoting $g_{\kappa}=\kappa V g$ we are led to the equation

$$
A_{\kappa}(v)=\frac{1}{2} v-K_{\kappa} v+V_{\kappa} F_{\kappa}(v)=g_{\kappa},
$$

where

$$
K_{\kappa}=\kappa K \frac{1}{\kappa}, \quad V_{\kappa}=\kappa V \kappa, \quad F_{\kappa}=\frac{1}{\kappa} F\left(\frac{1}{\kappa}\right) .
$$

We remark that the operator $A_{\kappa}$ is of the form needed in [14]. Appropriate choices for function spaces in order to make assumptions (C1)-(C5) in [14] hold are

$$
X^{0}=L^{2}(\Gamma), X=H^{\frac{1}{2}}(\Gamma), X^{*}=H^{-\frac{1}{2}}(\Gamma), Z=H^{1}(\Gamma) .
$$

Lemma 4.1. We have

(1) $K_{\kappa}: H^{\frac{1}{2}}(\Gamma) \rightarrow H^{1}(\Gamma)$ is bounded.

(2) $V_{\kappa}: H^{-\frac{1}{2}}(\Gamma) \rightarrow H^{\frac{1}{2}}(\Gamma)$ is an isomorphism and $V_{\kappa}: L^{2}(\Gamma) \rightarrow$ $H^{1}(\Gamma)$ is bounded.

(3) $F_{\kappa}: L^{2}(\Gamma) \rightarrow L^{2}(\Gamma)$ is bounded.

(4) $\left(A_{\kappa}(w)-A_{\kappa}\left(w^{\prime}\right) \mid\left(V_{\kappa}^{-1}\right)^{*}\left(w-w^{\prime}\right)\right)_{0} \geq c|| w-w^{\prime} \|_{H^{\frac{1}{2}}(\Gamma)}^{2}$. 
(5) $K_{\kappa}: L^{2}(\Gamma) \rightarrow H^{1}(\Gamma)$ is bounded.

Proof. We note that multiplication by regular functions $\kappa$ and $1 / \kappa$ defines an isomorphism from $H^{s}(\Gamma) \rightarrow H^{s}(\Gamma)$ for all $s$. Clearly $K_{\kappa}$ is a bounded linear operator from $H^{s}(\Gamma) \rightarrow H^{s+1}(\Gamma)$ for all $s$, which implies (1) and (5). The Lipschitz continuity of $F$ implies the Lipschitz continuity of $F_{\kappa}$ from $L^{2}(\Gamma) \rightarrow L^{2}(\Gamma)$. Thus (3) is valid. The properties (2) of $V_{\kappa}$ follow easily from the mapping properties of $V$. Finally, since the operator $A$ is strongly $V^{-1}$ monotone, we obtain

$$
\begin{aligned}
\left(A_{\kappa}(w)-\right. & \left.A_{\kappa}\left(w^{\prime}\right) \mid\left(V_{\kappa}^{-1}\right)^{*}\left(w-w^{\prime}\right)\right)_{0} \\
= & \left(\kappa\left(A\left(\frac{w}{\kappa}\right)-A\left(\frac{w^{\prime}}{\kappa}\right)\right) \mid\left(\frac{1}{\kappa} V^{-1} \frac{1}{\kappa}\right)^{*}\left(w-w^{\prime}\right)\right)_{0} \\
= & \left(A\left(\frac{w}{\kappa}\right)-A\left(\frac{w^{\prime}}{\kappa}\right) \mid V^{-1}\left(\frac{w}{\kappa}-\frac{w^{\prime}}{\kappa}\right)\right)_{0} \\
\geq & c\left\|\frac{w}{\kappa}-\frac{w^{\prime}}{\kappa}\right\|_{\frac{1}{2}}^{2} \geq c\left\|w-w^{\prime}\right\|_{\frac{1}{2}}^{2}
\end{aligned}
$$

which proves (4).

Let $v_{h} \in S_{h}^{d}$ be the collocation approximation of $v$ such that

$$
I_{h} A_{\kappa} v_{h}=I_{h} g_{\kappa}
$$

We have

Theorem 4.1. Assume $d>0$. Let $v \in H^{s}(\Gamma), 1 / 2<s \leq d+1$, be the solution of (4.4) and suppose that (A1), (A2) are valid. Then, for sufficiently small $h$, there exists a unique collocation solution such that

$$
\left\|v-v_{h}\right\|_{t} \leq c h^{s-t}\|v\|_{s},
$$

where $0 \leq t \leq s$ and $t<d+1 / 2$.

Proof. According to Lemma 4.1, the assumptions of [14] Theorem 2 are fulfilled and the estimate (4.7) follows by interpolation. 
Correspondingly, let $\tilde{v}_{h} \in S_{h}^{d}$ be the approximate collocation solution of $v$ such that

$$
\tilde{A}_{\kappa_{h}}\left(\tilde{v}_{h}\right):=\frac{1}{2} \tilde{v}_{h}-I_{h} K_{\kappa} \tilde{v}_{h}+I_{h} V_{\kappa} P_{h} F_{\kappa}\left(\tilde{v}_{h}\right)=I_{h} \kappa V g .
$$

Theorem 4.2. Let $d>0$. There exists a positive constant $c_{1}$ such that

$$
\left\|\tilde{A}_{\kappa_{h}}(\chi)-\tilde{A}_{\kappa_{h}}(\psi)\right\|_{\frac{1}{2}} \geq c_{1}\|\chi-\psi\|_{\frac{1}{2}}
$$

for all $\chi, \psi \in S_{h}^{d}$ when $0<h \leq h_{0}$. Moreover, equation (4.8) has a unique solution for $0<h \leq h_{0}$.

Theorem 4.3. Assume $d>0$. Let $v \in H^{s}(\Gamma), 1 / 2<s \leq d+1$ be the solution of (4.4), and suppose that the assumptions (A1), (A2) are valid. Then we have the estimates

$$
\begin{gathered}
\left\|\tilde{v}_{h}-v_{h}\right\|_{\frac{1}{2}} \leq c h^{s+\frac{1}{2}}\|v\|_{s}+c h^{\tau+\frac{1}{2}}\left\|F_{\kappa}(v)\right\|_{\tau} \\
\left\|v-\tilde{v}_{h}\right\|_{t} \leq c h^{s-t}|| v\left\|_{s}+c h^{\tau+1-\max \left(t, \frac{1}{2}\right)}\right\| F_{\kappa}(v) \|_{\tau},
\end{gathered}
$$

for $0 \leq t \leq s, t<d+1 / 2$, provided that $F_{\kappa}(v) \in H^{\tau}(\Gamma), 0 \leq \tau \leq d+1$.

The proofs of Theorems 4.2 and 4.3 are analogous to the proofs of Theorems 3.2 and 3.3, respectively. We remark that also Corollary 3.1 and Corollary 3.2 can be correspondingly extended to $v, v_{h}$ instead of $u, u_{h}$.

5. The effect of numerical integration. There is still the effect of numerical integration to be estimated. Here we assume that the mesh is uniform. Let $\mu$ be the characteristic function of the unit interval. The basis functions $\mu_{j}^{d}$ of the space $S_{h}^{d}$ are defined as translations $\mu_{j}^{d}(t)=\mu_{0}^{d}(t-j h)$, where $\mu_{0}^{d}$ is the 1-periodic extension of the $d$ fold convolution $\mu_{0}^{d}(t)=\mu^{d}(t / h):=(\mu * \cdots * \mu)(t / h), 0 \leq t \leq 1$. Let $K(\cdot, \cdot)$ be the kernel corresponding to the double layer operator and let 
$S(\cdot, \cdot)$ be the kernel of the smooth part of the single layer operator. We introduce the representations

$$
\tilde{v}_{h}=\sum_{j=0}^{N-1} \alpha_{j} \mu_{j}^{d}, \quad P_{h}\left(F\left(\frac{\tilde{v}_{h}}{\kappa}\right) \kappa\right)=\sum_{j=0}^{N-1} P_{h}\left(F\left(\frac{\tilde{v}_{h}}{\kappa}\right) \kappa\right)_{j} \mu_{j}^{d} .
$$

The approximate collocation problem (4.8) is equivalent to the following set of nonlinear equations:

$$
\begin{aligned}
& \frac{1}{2} \sum_{j=0}^{N-1} \alpha_{j} \mu_{j}^{d}\left(t_{i}\right)-\frac{h}{2 \pi} \kappa\left(t_{i}\right) \sum_{j=0}^{N-1} \alpha_{j} \int_{0}^{d+1} K\left(t_{i}, h \tau+h j\right) \mu^{d}(\tau) d \tau \\
& \quad-\frac{h}{2 \pi} \kappa\left(t_{i}\right) \sum_{j=0}^{N-1} P_{h}\left(F\left(\frac{\tilde{v}_{h}}{\kappa}\right) \kappa\right) j \int_{0}^{d+1} S\left(t_{i}, h \tau+h j\right) \mu^{d}(\tau) d \tau \\
& \quad-\frac{h}{2 \pi} \kappa\left(t_{i}\right) \sum_{j=0}^{N-1} P_{h}\left(F\left(\frac{\tilde{v}_{h}}{\kappa}\right) \kappa\right) j \int_{0}^{d+1} \mu^{d}(\tau) \ln \left|\left(\rho(i, j)+\tau-\frac{d+1}{2}\right) h\right| d \tau \\
& \quad=\kappa\left(t_{i}\right) \operatorname{Vg}\left(x\left(t_{i}\right)\right), \quad i=0,1, \ldots, N-1,
\end{aligned}
$$

where $\rho(i, j)=\min (|i-j|,|i-j+N|,|i-j-N|)$. By using the notation

$$
\begin{aligned}
& k_{i j}=h \int_{0}^{d+1} K\left(t_{i}, h \tau+h j\right) \mu^{d}(\tau) d \tau \\
& s_{i j}=s_{i j}^{0}+s_{i j}^{1} ; s_{i j}^{0}=h \int_{0}^{d+1} \mu^{d}(\tau) \ln \left|\left(\rho(i, j)+\tau-\frac{d+1}{2}\right) h\right| d \tau, \\
& s_{i j}^{1}=h \int_{0}^{d+1} S\left(t_{i}, h \tau+h j\right) \mu^{d}(\tau) d \tau,
\end{aligned}
$$

the nonlinear system can be rewritten as

$$
\begin{gathered}
\frac{1}{2} \sum_{j=0}^{N-1} \alpha_{j} \mu_{j}^{d}\left(t_{i}\right)-\frac{\kappa\left(t_{i}\right)}{2 \pi} \sum_{j=0}^{N-1} \alpha_{j} k_{i j}-\frac{\kappa\left(t_{i}\right)}{2 \pi} \sum_{j=0}^{N-1} P_{h}\left(F\left(\frac{\tilde{v}_{h}}{\kappa}\right) \kappa\right)_{j} s_{i j}^{1} \\
-\frac{\kappa\left(t_{i}\right)}{2 \pi} \sum_{j=0}^{N-1} P_{h}\left(F\left(\frac{\tilde{v}_{h}}{\kappa}\right) \kappa\right)_{j} s_{i j}^{0} \\
=\kappa\left(t_{i}\right) V g\left(x\left(t_{i}\right)\right), \quad i=0, \ldots, N-1 .
\end{gathered}
$$


We replace the integrals by numerical quadratures,

$$
\tilde{k}_{i j}=h \sum_{l} \beta_{l} K\left(t_{i}, \tau_{j l}\right), \quad \tilde{s}_{i j}^{1}=h \sum_{l} \beta_{l} S\left(t_{i}, \tau_{j l}\right)
$$

where the numbers $\beta_{l}$ are weights and the points $\tau_{j l}$ are corresponding abscissae of the numerical quadrature rule. We suppose that the quadrature satisfies

$$
\left|k_{i j}-\tilde{k}_{i j}\right| \leq c h^{\sigma}, \quad\left|s_{i j}^{1}-\tilde{s}_{i j}^{1}\right| \leq c h^{\sigma} .
$$

For the right hand side of (5.2) we use the orthogonal projection approximation $\kappa V g\left(x_{i}\right) \approx \kappa V P_{h} g\left(x_{i}\right)$. The resulting set of nonlinear equations is

$$
\begin{gathered}
\frac{1}{2} \sum_{j=0}^{N-1} \hat{\alpha}_{j} \mu_{j}^{d}\left(t_{i}\right)-\frac{\kappa\left(t_{i}\right)}{2 \pi} \sum_{j=0}^{N-1} \hat{\alpha}_{j} \tilde{k}_{i j}-\frac{\kappa\left(t_{i}\right)}{2 \pi} \sum_{j=0}^{N-1} P_{h}\left(F\left(\frac{\hat{v}_{h}}{\kappa}\right) \kappa\right)_{j} \tilde{s}_{i j}^{1} \\
-\frac{\kappa\left(t_{i}\right)}{2 \pi} \sum_{j=0}^{N-1} P_{h}\left(F\left(\frac{\hat{v}_{h}}{\kappa}\right) \kappa\right)_{j} s_{i j}^{0} \\
=\kappa\left(t_{i}\right) V P_{h} g\left(x_{i}\right), \quad i=0,1, \ldots, N-1 .
\end{gathered}
$$

The system (5.4) defines the mapping $\hat{A}_{\kappa_{h}}: S_{h}^{d} \rightarrow S_{h}^{d}$ such that

$$
\hat{A}_{\kappa_{h}}\left(\hat{v}_{h}\right)=I_{h} \kappa V P_{h} g
$$

We deduce the solvability of (5.5) from the stability and continuity of the operator $\hat{A}_{\kappa_{h}}$.

Theorem 5.1. Let $d>0$. There exists a positive constant $c_{2}$ such that

$$
\left\|\hat{A}_{\kappa_{h}}(\chi)-\hat{A}_{\kappa_{h}}(\psi)\right\|_{\frac{1}{2}} \geq c_{2}\|\chi-\psi\|_{\frac{1}{2}}
$$

for all $\chi, \psi \in S_{h}^{d}$ when $0<h \leq h_{0}$. Moreover, equation (5.5) has a unique solution for $0<h \leq h_{0}$. 
Proof. By using the $\left(V_{\kappa}^{-1}\right)^{*}$-stability of the operator $\tilde{A}_{\kappa_{h}}$ we have (5.7)

$$
\begin{aligned}
& \left(\hat{A}_{\kappa_{h}}(\chi)-\hat{A}_{\kappa_{h}}(\psi) \mid\left(V_{\kappa}^{-1}\right)^{*}(\chi-\psi)\right)_{0} \\
& =\left(\tilde{A}_{\kappa_{h}}(\chi)-\tilde{A}_{\kappa_{h}}(\psi) \mid\left(V_{\kappa}^{-1}\right)^{*}(\chi-\psi)\right)_{0} \\
& \quad+\left(\left(\hat{A}_{\kappa_{h}}-\tilde{A}_{\kappa_{h}}\right)(\chi)-\left(\hat{A}_{\kappa_{h}}-\tilde{A}_{\kappa_{h}}\right)(\psi) \mid\left(V_{\kappa}^{-1}\right)^{*}(\chi-\psi)\right)_{0} \\
& \geq c\|\chi-\psi\|_{\frac{1}{2}}\left\|\left(V_{\kappa}^{-1}\right)^{*}(\chi-\psi)\right\|_{-\frac{1}{2}} \\
& \quad-c\left\|\left(\hat{A}_{\kappa_{h}}-\tilde{A}_{\kappa_{h}}\right)(\chi)-\left(\hat{A}_{\kappa_{h}}-\tilde{A}_{\kappa_{h}}\right)(\psi)\right\|_{\frac{1}{2}}\left\|\left(V_{\kappa}^{-1}\right)^{*}(\chi-\psi)\right\|_{-\frac{1}{2}}
\end{aligned}
$$

for all splines $\chi, \psi$. For further estimation, we compare the operators $\tilde{A}_{\kappa_{h}}$ and $\hat{A}_{\kappa_{h}}$ componentwise. With $\chi=\sum_{j=0}^{N-1} \alpha_{j} \mu_{j}^{d}$, we have

$$
\begin{aligned}
\left(\tilde{A}_{\kappa_{h}}(\chi)\right)_{i}= & \frac{\alpha_{i}}{2}-\frac{\kappa\left(t_{i}\right)}{2 \pi} \sum_{j=0}^{N-1} k_{i j} \alpha_{j}-\frac{\kappa\left(t_{i}\right)}{2 \pi} \sum_{j=0}^{N-1} s_{i j}^{1} P_{h}\left(F\left(\frac{\chi}{\kappa}\right) \kappa\right)_{j} \\
& -\frac{\kappa\left(t_{i}\right)}{2 \pi} \sum_{j=0}^{N-1} s_{i j}^{0} P_{h}\left(F\left(\frac{\chi}{\kappa}\right) \kappa\right)_{j}, \\
\left(\hat{A}_{\kappa_{h}}(\chi)\right)_{i}= & \frac{\alpha_{i}}{2}-\frac{\kappa\left(t_{i}\right)}{2 \pi} \sum_{j=0}^{N-1} \tilde{k}_{i j} \alpha_{j}-\frac{\kappa\left(t_{i}\right)}{2 \pi} \sum_{j=0}^{N-1} \tilde{s}_{i j}^{1} P_{h}\left(F\left(\frac{\chi}{\kappa}\right) \kappa\right)_{j} \\
& -\frac{\kappa\left(t_{i}\right)}{2 \pi} \sum_{j=0}^{N-1} s_{i j}^{0} P_{h}\left(F\left(\frac{\chi}{\kappa}\right) \kappa\right)_{j} .
\end{aligned}
$$

Writing $\psi=\sum_{j=0}^{N-1} \omega_{j} \mu_{j}^{d}$, we obtain

$$
\begin{array}{r}
\left(\left(\hat{A}_{\kappa_{h}}-\tilde{A}_{\kappa_{h}}\right)(\chi)\right)_{i}-\left(\left(\hat{A}_{\kappa_{h}}-\tilde{A}_{\kappa_{h}}\right)(\psi)\right)_{i}=\frac{\kappa\left(t_{i}\right)}{2 \pi} \sum_{j=0}^{N-1}\left(\tilde{k}_{i j}-k_{i j}\right)\left(\alpha_{j}-\omega_{j}\right) \\
+\frac{\kappa\left(t_{i}\right)}{2 \pi} \sum_{j=0}^{N-1}\left(\tilde{s}_{i j}^{1}-s_{i j}^{1}\right)\left\{P_{h}\left(F\left(\frac{\chi}{\kappa}\right) \kappa\right)_{j}-P_{h}\left(F\left(\frac{\psi}{\kappa}\right) \kappa\right)_{j}\right\}
\end{array}
$$

The norm equivalence [5]

$$
c_{1}\|\chi\|_{0} \leq\left\{h \sum_{j=0}^{N-1} \alpha_{j}^{2}\right\}^{\frac{1}{2}} \leq c_{2}\|\chi\|_{0}, \quad \chi \in S_{h}^{d}
$$


gives the estimate

(5.11)

$$
\begin{aligned}
& \left\|\left(\hat{A}_{\kappa_{h}}-\tilde{A}_{\kappa_{h}}\right)(\chi)-\left(\hat{A}_{\kappa_{h}}-\tilde{A}_{\kappa_{h}}\right)(\psi)\right\|_{0} \\
& \leq c\left\{h \sum_{i=0}^{N-1}\left[\sum_{j=0}^{N-1}\left(\tilde{k}_{i j}-k_{i j}\right)\left(\alpha_{j}-\omega_{j}\right)\right]^{2}\right\}^{\frac{1}{2}} \\
& +c\left\{h \sum_{i=0}^{N-1}\left[\sum_{j=0}^{N-1}\left(\tilde{s}_{i j}^{1}-s_{i j}^{1}\right)\left\{P_{h}\left(F\left(\frac{\chi}{\kappa}\right) \kappa\right)_{j}-P_{h}\left(F\left(\frac{\psi}{\kappa}\right) \kappa\right)_{j}\right\}_{j}\right]^{2}\right\}^{\frac{1}{2}} \\
& \leq c\left\{\sum_{i=0}^{N-1} \sum_{j=0}^{N-1}\left(\tilde{k}_{i j}-k_{i j}\right)^{2}\right\}^{\frac{1}{2}}\left\{h \sum_{j=0}^{N-1}\left(\alpha_{j}-\omega_{j}\right)^{2}\right\}^{\frac{1}{2}} \\
& \quad+c\left\{\sum_{i=0}^{N-1} \sum_{j=0}^{N-1}\left(\tilde{s}_{i j}^{1}-s_{i j}^{1}\right)^{2}\right\}^{\frac{1}{2}}\left\{h \sum_{j=0}^{N-1}\left[P_{h}\left\{F\left(\frac{\chi}{\kappa}\right) \kappa-F\left(\frac{\psi}{\kappa}\right) \kappa\right\}_{j}\right]^{2}\right\}^{\frac{1}{2}} \\
& \leq c h^{\sigma-1}\left(\|\chi-\psi\|_{0}+\left\|\left(F\left(\frac{\chi}{\kappa}\right)-F\left(\frac{\psi}{\kappa}\right)\right) \kappa\right\|_{0}\right) \\
& \leq c h^{\sigma-1}\|\chi-\psi\|_{0} .
\end{aligned}
$$

The stability estimate (5.6) follows from (5.7) combined with (5.11), (2.2) and the Schwarz inequality. Existence of the solution as well as uniqueness are proved as in Theorem 3.2.

The following consistency estimate is valid.

Lemma 5.1. We have

$$
\begin{aligned}
\left\|\left(\tilde{A}_{\kappa_{h}}-\hat{A}_{\kappa_{h}}\right)(\chi)\right\|_{0} \leq & c\left[\sum_{i=0}^{N-1} \sum_{j=0}^{N-1}\left|k_{i j}-\tilde{k}_{i j}\right|^{2}\right]^{\frac{1}{2}}\|\chi\|_{0} \\
& +c\left[\sum_{i=0}^{N-1} \sum_{j=0}^{N-1}\left|s_{i j}^{1}-\tilde{s}_{i j}^{1}\right|^{2}\right]^{\frac{1}{2}}\left\|P_{h}\left(F\left(\frac{\chi}{\kappa}\right) \kappa\right)\right\|_{0} \\
\leq & c h^{\sigma-1}\left(\|\chi\|_{0}+\left\|F\left(\frac{\chi}{\kappa}\right) \kappa\right\|_{0}\right)
\end{aligned}
$$


for all $\chi \in S_{h}^{d}$. Furthermore,

$$
\left\|\left(I_{h} \kappa V g-I_{h} \kappa V P_{h} g\right)\right\|_{0} \leq c h^{s}\|g\|_{s-1}
$$

for any $g \in H^{s-1}(\Gamma), 1 / 2<s \leq d+1$.

Proof. As in the proof of the previous theorem (cf. (5.11)), we obtain

$$
\left\|\left(\tilde{A}_{\kappa_{h}}-\hat{A}_{\kappa_{h}}\right)(\chi)\right\|_{0} \leq\left\|T_{1}(\chi)\right\|_{0}+\left\|T_{2}(\chi)\right\|_{0}
$$

where

$$
\begin{aligned}
\left\|T_{2}(\chi)\right\|_{0} & \leq c\left\{h\left[\sum_{i=0}^{N-1} \sum_{j=0}^{N-1}\left(s_{i j}^{1}-\tilde{s}_{i j}^{1}\right)^{2}\right]\left[\sum_{j=0}^{N-1}\left(P_{h}\left(F\left(\frac{\chi}{\kappa}\right) \kappa\right)_{j}\right)\right]^{2}\right\}^{\frac{1}{2}} \\
& \leq c\left[\sum_{i=0}^{N-1} \sum_{j=0}^{N-1}\left(s_{i j}^{1}-\tilde{s}_{i j}^{1}\right)^{2}\right]^{\frac{1}{2}}\left\{h\left[\sum_{j=0}^{N-1}\left(P_{h}\left(F\left(\frac{\chi}{\kappa}\right) \kappa\right)_{j}\right)^{2}\right]\right\}^{\frac{1}{2}} \\
& \leq c\left[\sum_{i=0}^{N-1} \sum_{j=0}^{N-1}\left(s_{i j}^{1}-\tilde{s}_{i j}^{1}\right)^{2}\right]^{\frac{1}{2}}\left\|P_{h}\left(F\left(\frac{\chi}{\kappa}\right) \kappa\right)\right\|_{0} \\
& \leq c h^{\sigma-1}\left\|F\left(\frac{\chi}{\kappa}\right) \kappa\right\|_{0} .
\end{aligned}
$$

Analogously, we have $\left\|T_{1}(\chi)\right\|_{0} \leq c h^{\sigma-1}\|\chi\|_{0}$. Thus, (5.12) is valid. The estimate (5.13) follows directly from the approximation properties (3.3), (3.7) and from the mapping properties of $V$.

Having found the spline approximation $\hat{v}_{h}$ of $v=\kappa u$, we define for $u=(1 / \kappa) v$ the (nonspline) approximation by setting $\hat{u}_{h}=(1 / \kappa) \hat{v}_{h}$. We summarize all convergence results in the following theorem.

Theorem 5.2. Assume $d>0$. Let $g \in H^{s-1}(\Gamma)$ and let $v \in H^{s}(\Gamma)$, $1 / 2<s \leq d+1$ be the solution of (4.4) and suppose that the assumptions 
(A1), (A2) are valid. Then we have the estimates

$$
\begin{aligned}
\left\|\tilde{v}_{h}-\hat{v}_{h}\right\|_{\frac{1}{2}} \leq & c h^{s-\frac{1}{2}}\|g\|_{s-1}+c h^{\sigma-\frac{3}{2}}\left(\|v\|_{\frac{1}{2}}+\|F(0)\|_{0}\right) \\
\left\|u-\hat{u}_{h}\right\|_{t} \leq & c\left\|v-\hat{v}_{h}\right\|_{t} \leq c h^{s-t}\left(\|v\|_{s}+\|g\|_{s-1}\right) \\
& +c h^{\tau+1-\max \left(t, \frac{1}{2}\right)}\left\|F_{\kappa}(v)\right\|_{\tau} \\
& +c h^{\sigma-1-\max \left(t, \frac{1}{2}\right)}\left(\|v\|_{\frac{1}{2}}+\|F(0)\|_{0}\right),
\end{aligned}
$$

for $0 \leq t \leq s, t<d+1 / 2$, provided that $F_{\kappa}(v) \in H^{\tau}(\Gamma), 0 \leq \tau \leq d+1$.

Proof. Stability and consistency imply convergence and the order of convergence is at least the order of consistency. Since the identities $\tilde{A}_{\kappa_{h}}\left(\tilde{v}_{h}\right)=I_{h} \kappa V g$ and $\hat{A}_{\kappa_{h}}\left(\hat{v}_{h}\right)=I_{h} \kappa V P_{h} g$ are valid, we have by (5.12), $(5.13)$

$$
\begin{aligned}
\left\|\hat{v}_{h}-\tilde{v}_{h}\right\|_{\frac{1}{2}} & \leq c\left\|\hat{A}_{\kappa_{h}}\left(\hat{v}_{h}\right)-\hat{A}_{\kappa_{h}}\left(\tilde{v}_{h}\right)\right\|_{\frac{1}{2}} \\
& \leq c\left\|\mid I_{h} \kappa V\left(P_{h}-I\right) g\right\|_{\frac{1}{2}}+\left\|\left(\tilde{A}_{\kappa_{h}}-\hat{A}_{\kappa_{h}}\right)\left(\tilde{v}_{h}\right)\right\|_{\frac{1}{2}} \\
& \leq c h^{s-\frac{1}{2}}\|g\|_{s-\frac{1}{2}}+c h^{\sigma-\frac{3}{2}}\left(\|v\|_{\frac{1}{2}}+\|F(0)\|_{0}\right) .
\end{aligned}
$$

Thus, (5.15) is proved. Since

$$
\left\|u-\hat{u}_{h}\right\|_{t} \leq c\left\|v-\hat{v}_{h}\right\|_{t}
$$

the estimate (5.16) follows from Theorem 4.3 combined with (5.15) and $(2.2)$.

6. Numerical results. Finally, we present some numerical results in order to illustrate our asymptotic convergence estimates. First, we consider the following nonlinear integral equations of type $A(u)=V g$ on the boundary of a disk of radius $r$. We denote $F_{a b c}(u)=a u+b u^{3}+$ $c u^{5},|u| \leq M$, where $a, b, c$ are real numbers such that $F_{a b c}$ is monotone. We define the nonlinearity

$$
F(u)= \begin{cases}F_{a b c}(u), & \text { if }|u| \leq M \\ F_{a b c}(M)+F_{a b c}^{\prime}(M)(u-M), & \text { if } u>M \\ F_{a b c}(M)+F_{a b c}^{\prime}(-M)(u-M), & \text { if } u<-M\end{cases}
$$


and the right side $V g=g_{a b c}$ by the formula

$$
\begin{aligned}
g_{a b c}(t)=\left[\frac{4+4 a r+3 b r+5 c r}{8}\right] \cos (2 \pi t) \\
+\left[\frac{b r}{24}+\frac{5 c r}{96}\right] \cos (6 \pi t)+\frac{c r}{160} \cos (10 \pi t) .
\end{aligned}
$$

Then the function $u(t)=\cos (2 \pi t)$ is the solution of the nonlinear integral equation (1.2). Numerical solution leads to a system of nonlinear equations which is solved with the Newton method. We use piecewise linear approximations. For numerical integration, we apply the low order spline-weighted grid-point rule with three points in the reference interval. This formula gives the accuracy $\sigma=5[\mathbf{1}]$. The results in the following table are typical of this family of model problems. For nonzero values of $c$, the table remains essentially the same. Only the number of Newton iterations will increase.

TABLE 1.

\begin{tabular}{|c|c|c|}
\hline \multicolumn{3}{|c|}{$a=1.0, b=1.0, c=0.0, r=0.4$} \\
\hline Nodes & $L^{2}$-error & Rate \\
\hline 16 & 0.009149 & \\
& & 2.004 \\
32 & 0.002281 & \\
& & 2.003 \\
64 & 0.000569 & \\
\hline
\end{tabular}

Our second example is taken from [4]. Here we have the nonlinear potential problem (1.1) with $g(x, u)=u+\sin (u)$. The function $f$ is chosen such that the true solution will be $u_{0}(x, y)=e^{x} \cos (y)$. We use the elliptical region $\Omega=\left\{x \mid(x / a)^{2}+(y / b)^{2}<1\right\}$ with the values $a=1$ and $b=2$. In the following table we give the $L^{2}$-error for the boundary density. 
TABLE 2 .

\begin{tabular}{|c|c|c|}
\hline \multicolumn{3}{|c|}{$a=1.0, b=2.0$} \\
\hline Nodes & $L^{2}$-error & Rate \\
\hline 16 & 0.1531 & \\
& & 1.82 \\
32 & 0.0434 & \\
& & 1.95 \\
\hline
\end{tabular}

In both examples the convergence measured with respect to $L^{2}$-norm is quadratic. Finally, we remark that the use of spline-weighted gridpoint rule corresponding to the accuracy $\sigma=3$ also gives the quadratic convergence with respect to $L^{2}$-norm. In this case our theoretical rate of convergence is $3 / 2$.

Acknowledgment. The authors would like to express their gratitude to Prof. K.E. Atkinson and the referee for their suggestions to improve the original manuscript of this article.

\section{REFERENCES}

1. D.N. Arnold and W.L. Wendland, Collocation versus Galerkin procedures for boundary integral methods, in Boundary element methods in engineering, Springer Verlag, Berlin, Heidelberg, New York, 1982, 18-38.

2. - On the asymptotic convergence of collocation methods, Math. Comp. 41 (1983), 349-381.

3. - The convergence of spline collocation for strongly elliptic equations on curves, Numer. Math. 47 (1985), 317-343.

4. K.E. Atkinson and G. Chandler, BIE methods for solving Laplace equation with nonlinear boundary conditions: The smooth boundary case, Math. Comp. 55 (1990), 451-472.

5. J.P. Aubin, Approximation of elliptic boundary value problems, John Wiley \& Sons, Inc., New York, 1972.

6. K. Deimling, Nonlinear functional analysis, Springer Verlag, Berlin, Heidelberg, New York, Tokyo, 1985.

7. J. Elschner and G. Schmidt, On spline interpolation in periodic Sobolev spaces, preprint 01/83, Dept. Math. Akademie der Wissenschaften der DDR, Berlin (1985). 
8. G.C. Hsiao and W.L. Wendland, A finite element method for some integral equations of the first kind equations on curves, J. Math. Anal. Appl. 58 (1977), 449-481.

9. D. Pascali and S. Sburlan, Nonlinear mappings of monotone type, Siijthoff and Noordhoff International Publishers, Bucharest, 1978.

10. K. Ruotsalainen and J. Saranen, A dual method to the collocation method, Math. Meth. in Applied Sci. 10 (1988), 439-445.

11. - On the convergence of the Galerkin method for nonsmooth solutions of integral equations, Numer. Math. 54 (1988), 295-302.

12. - On the collocation method for a nonlinear boundary integral equation, J. Comput. Appl. Math 28 (1989), 339-348.

13. K. Ruotsalainen and W.L. Wendland, On the boundary element method for some nonlinear boundary value problems, Numer. Math. 53 (1988), 299-314.

14. J. Saranen, Projection methods for a class of Hammerstein equations, SIAM J. Numer. Anal. 27 (1990), 1445-1449.

Section of Mathematics, Faculty of Technology, University of Oulu, SF-90570 OUlu, Finland 